\title{
Modelling the association of disability according to the WHO International Classification of Functioning, Disability and Health (ICF) with mortality in the British Women's Heart and Health Study
}

\author{
Caroline Dale, ${ }^{1}$ David Prieto-Merino, ${ }^{2}$ Hannah Kuper,' Joy Adamson, ${ }^{3}$ Ann Bowling, ${ }^{5}$ \\ Shah Ebrahim, ${ }^{1,6}$ Juan P Casas ${ }^{1,7}$
}

- Additional appendices are published online only. To view these files please visit the journal online (http://jech.bmj. com/content/66/2.toc).

${ }^{1}$ Department of

Non-communicable Disease Epidemiology, Faculty of Epidemiology and Population Health, London School of Hygiene and Tropical Medicine, UK

${ }^{2}$ Department of Medical Statistics, Faculty of Epidemiology and Population Health, London School of Hygiene and Tropical Medicine, UK

${ }^{3}$ Clinical Research Department,

Faculty of Infectious and

Tropical Diseases, London

School of Hygiene and Tropical

Medicine, UK

${ }^{4}$ Department of Health

Sciences, University of York, UK

${ }^{5}$ Faculty of Health and Social

Care Sciences, Kingston

University and St George's,

University of London, UK

${ }^{6}$ South Asia Network for Chronic

Disease, Public Health

Foundation of India, New Delhi, India

${ }^{7}$ Department of Epidemiology and Public Health, University College London, UK

\section{Correspondence to}

Caroline Dale, Faculty of

Epidemiology and Population

Health, London School of

Hygiene and Tropical Medicine,

UK; caroline.dale@Ishtm.ac.uk

Caroline Dale, David Prieto-Merino contributed equally to this work.

Accepted 10 August 2011 Published Online First 19 October 2011

\section{ABSTRACT \\ Background The WHO International Classification of Functioning, Disability and Health (ICF) is now the dominant model for exploring the social consequences of a health condition. This paper investigates the association of the different ICF disability domains with mortality.}

Methods Data are from the British Women Heart and Health Study, a large $(n=4157)$ prospectively studied cohort of women randomly selected from 23 towns aged 64-83 years in 2003. Scores were calculated to describe the cumulative load of impairments, limitations and restrictions within each ICF domain. Cox proportional hazards regression was used to calculate mortality HRs per unit score increase within each ICF domain. Adjustments were made for age, town, living status, socioeconomic status, lifestyle behaviours and health conditions.

Results Each ICF domain was associated with mortality after controlling for lifestyle factors and health conditions. However, only complex activities $(\mathrm{HR}=1.09$, $95 \% \mathrm{Cl} 1.01$ to 1.18$)$ and participation $(\mathrm{HR}=1.10,95 \%$ $\mathrm{Cl} 1.04$ to 1.16 ) were independent predictors of mortality following adjustment for all other disability domains and potential confounders.

Conclusions Results suggest that difficulties with complex activities or social participation could be used to identify and target women at high risk of dying. Interventions to facilitate complex activities or improve social participation may help to delay mortality in elderly women.

\section{INTRODUCTION}

Projections suggest that the proportion of the population aged above 65 years will increase from $16 \%$ of the population in 2008 to $23 \%$ by $2030 .{ }^{1}$ Assuming no improvement in age-specific disability rates, the number of older disabled people in the UK is projected to increase by $40 \%$ between 2002 and $2022 .^{2}$ An alternative scenario is that extra years of life could be lived in better health with morbidity and disability postponed to ever-older ages-the compression of morbidity theory. ${ }^{3}$ Indeed, recent UK government policy has been consistently targeted at helping people to live longer and to live more healthily. ${ }^{4-6} \mathrm{~A}$ third 'steady-state' scenario is that both the number of years lived in good health and the number of years lived with disability will increase. $^{7}$
It currently remains unclear which scenario is most probable. $^{8}$ In the USA, some evidence supports the compression of morbidity theory. ${ }^{9}$ UK statistics suggest that recent improvements in life expectancy at age 65 have not been matched by improvements in disability-free life expectancy. ${ }^{10}$ Other evidence suggests that more recent UK cohorts may have a higher level of disability, contrasting with declining trends in other countries. ${ }^{11} 12$ Severity of disability may also influence evidence supporting the different scenarios. For example, in England and Wales, increasing prevalence of limiting longstanding illness may be due to an increase in more minor problems while the prevalence of the most severe disabilities is declining. ${ }^{13}$ Hence, a better understanding of the implications of different conceptualisations of disability for mortality is needed.

The medical model of disability attributes limitations in activity and participation solely to various physical conditions. In contrast, the social model of disability recognises that these are the product both of the individual's physical capacity and the environment they inhabit. ${ }^{14}$ The social model is incorporated into the WHO's International Classification of Functioning, Disability and Health (ICF) (figure 1), now the dominant model for exploring the consequences of a health condition on disability. ${ }^{15} 16$ According to the ICF, disability is described as functioning according to three domains: impairments, activities and participation. Impairment relates to pathological body function and structural changes (eg, visual impairment), activities describe the capacity of a person to carry out basic (eg, drinking from a cup) and complex tasks (eg, preparing a hot meal), while participation refers to the extent of involvement in life situations (eg, going on outings). These three domains are placed within the context of environment and personal factors.

In epidemiological studies of the effects of disability on mortality, many studies of isolated aspects of functioning have been undertaken, often limited to walking or activities of daily living. ${ }^{17-19}$ Thus, the majority of studies to date relate to what would be classed as impairments or activities according to ICF. However, a large meta-analysis has recently highlighted the importance of social relationships for survival, with effects comparable in size to established risk factors (smoking, alcohol, physical inactivity). ${ }^{20}$ To our knowledge, no study 
Health condition

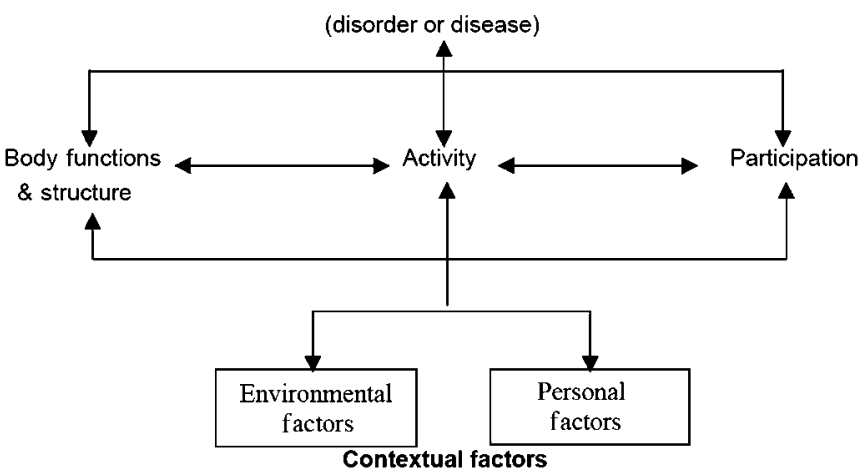

Figure 1 The International Classification of Functioning, Disability and Health (ICF). Source: World Health Organization Geneva 2002, 'Towards a Common Language for Functioning, Disability and Health: ICF'.

has attempted to explicitly contrast the effect of different ICF domains of disability on mortality. Existing studies are also diverse with respect to populations (nationality, community based, in-patients, gender), with the study of disability in older women particularly under-represented.

We examined the association between disability and mortality to gain a better understanding of the optimal targets for health and social care interventions to reduce mortality in older people. The independent effects of disability domains defined according to the ICF on all-cause mortality were compared using data from a large nationally representative cohort of older women.

\section{METHODS}

\section{Study design}

The British Women's Heart and Health Study (BWHHS) is a prospective cohort of elderly women recruited in 1999 from General Practitioner (GP) practices in 23 towns across England, Scotland and Wales. Women aged 60-79 years were selected at random from GP registers in these towns. Four thousand two hundred and eighty-six of the women originally invited agreed to participate in the study, corresponding to a response rate of $59 \%$. There have been two subsequent follow-ups of the BWHHS in 2003 and 2007 (with a further follow-up in progress). In this study, the 2003 wave was taken as baseline since disability was much more comprehensively measured at this follow-up. Of the 4286 women recruited in 1999, data for 4132 women $(96 \%)$ were available in 2003. Ethics approval for the BWHHS has been granted by the 23 Local Research Ethics Committees for the study population and the London School of Hygiene \& Tropical Medicine Ethics Committee. All women gave signed informed consent at baseline.

\section{Outcome}

Women were prospectively followed until February 2010 with deaths recorded via the NHS Central Registry. Six hundred and fifty-three deaths were recorded during the 7-year period of follow-up, with the number of deaths per year shown in table 1 . The proportion of women who died during follow-up was 0.158 .

\section{Exposure variables}

All variables in the 2003 wave of BWHHS were assessed for inclusion in the ICF framework. This process resulted in the identification of 40 variables: 8 impairments, 24 activities and 8 participations (online appendix A). These included variables from the EQ-5D, Nottingham Health Profile Part II, activities of
Table 1 Survival status by year

\begin{tabular}{llcll}
\hline Year & Population at risk & Number of deaths & $\begin{array}{l}\text { Survival } \\
\text { proportion }\end{array}$ & 95\% Cl \\
\hline 0 & 4132 & 0 & 1.00 & 1.00 to 1.00 \\
1 & 4068 & 65 & 0.98 & 0.98 to 0.99 \\
2 & 3979 & 88 & 0.96 & 0.96 to 0.97 \\
3 & 3884 & 95 & 0.94 & 0.93 to 0.95 \\
4 & 3789 & 95 & 0.92 & 0.91 to 0.93 \\
5 & 3694 & 95 & 0.89 & 0.89 to 0.90 \\
6 & 3581 & 114 & 0.87 & 0.86 to 0.88 \\
7 & 3479 & 101 & 0.84 & 0.83 to 0.85 \\
\hline
\end{tabular}

daily living and instrumental activities of daily living. ${ }^{21-23}$ The authors (CD and HK) consulted the ICF browser to provide objective classification of the variables into the different ICF domains of health conditions, impairments, activities or participation. $^{24}$

The ICF browser distinguishes impairments from activities and participation but does not clearly distinguish between activities and participation. ${ }^{24}$ Independent external expert opinion was sought for any items where uncertainty remained. Activities were further subdivided into basic and complex activities. Basic activities were defined as simple actions to accomplish a task (eg, bending down) and complex activities as those involving several faculties and cognition (eg, preparing a hot meal). Using this definition, the authors (CD and HK) classified the 24 activities into 16 basic and 8 complex activities (online appendix A). No item was assigned to more than one ICF domain.

Health conditions were categorised into two groups depending on whether they related to cardiovascular disease or not. Cardiovascular health conditions included heart attack, heart failure, angina, stroke, deep vein thrombosis, claudication, pulmonary embolism, diabetes and aortic aneurysm. Noncardiovascular health conditions included cancer, asthma, bronchitis, gastric, peptic or duodenal ulcer, gout, gall bladder disease, osteoporosis, arthritis, cataract and glaucoma. The cumulative load of adverse conditions within each group was summed.

The cumulative number of adverse lifestyle factors was also summed. Lifestyle factors included smoking (1=current smoker), drinking alcohol ( $1=$ most days), healthy diet $(1=$ fruit and vegetables less often than four or five times per day) and physical inactivity ( $1=$ less than $2 \mathrm{~h}$ moderate or vigorous activity per week).

Scores were calculated to express the cumulative load of disabilities within each ICF domain (impairments, activities, participation) and also for health conditions and lifestyle factors. All variables were binary $(0 / 1)$ coded such that $1=$ adverse. To account for missing data, participants were assigned a score based on the proportion of adverse endorsements to the number of questions answered in the domain. The proportion was subsequently rescaled from 0 to 10 (online appendix B), so 1 point of the score reflects a $10 \%$ increase in the proportion of endorsement. In all domains, a higher score indicates a greater adverse load.

There are a number of potential confounders of the disability-mortality association, including age, marital status, social support and socioeconomic status (SES). ${ }^{25-31}$ Hence, age (10 categories), town (23 towns), living status (living alone or with other people) and SES were considered to be potential confounders. SES was measured as a continuous score of lifecourse adversity where a higher score indicates more adverse events. ${ }^{32}$ Living status was included as a succinct measure of both marital status and social support. 


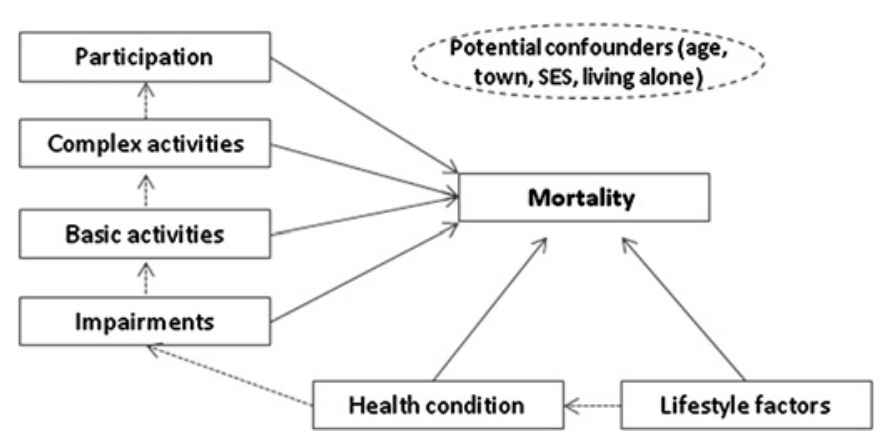

Figure 2 Hypothetical conceptual framework for the association of lifestyle factors, comorbidities and domains of disability with mortality. SES, socioeconomic status.

\section{STATISTICAL ANALYSES}

A hypothetical framework of the association of ICF domains plus health conditions and lifestyle factors with mortality was used to inform the statistical modelling (figure 2). The independent association of each ICF domain with mortality was estimated and subsequently adjusted for confounders, lifestyle factors, health conditions and other ICF domains. Adjustments were made according to the following sequence:

Model 0: Crude association.

Model 1: Adjusted for potential confounders only.

Model 2: Adjusted for potential confounders, lifestyle factors, health conditions and prior ICF domains.

Model 3: Adjusted for potential confounders, lifestyle factors health conditions and subsequent ICF domains (but not for prior domains).

Model 4: Simultaneous adjustment for potential confounders, lifestyle factors, health conditions and all other ICF domains.

In each of the models above, Cox proportional hazards regression was used to calculate the increase in mortality HR per unit of disability score within each domain. HRs therefore represent the increased mortality hazard per $10 \%$ increase in the proportion of endorsements within a domain.

\section{RESULTS}

The response profile for each disability domain, lifestyle factors, health conditions and confounders is described in table 2 according to mortality status at end of follow-up. Response to impairments, activities, participation, SES and living alone was lower than other variables in both follow-up categories. Baseline response rates were consistently higher in women who were alive at the end of follow-up compared with those who died during follow-up. These differences in response by mortality status were especially marked for impairments, activities, participation and living status. Mean scores were consistently higher among those who subsequently died for all domains and confounding variables, indicating higher adverse load in those who died.

Histograms (online appendix C) illustrate score frequency within each domain and the trend in the probability of dying with $95 \%$ CIs by score. There was a consistent trend for higher probability of dying with increasing disability score, with similar trends for lifestyle factors and health conditions.

Figure 3 illustrates the increase in mortality hazard per unit increase in disability domain score. Unadjusted HRs (models 0; figure 3 ) were similar in all disability domains ranging from 1.17 (95\% CI 1.14 to 1.20 ) for basic activities to 1.21 (95\% CI 1.18 to 1.24) for complex activities. Compared with unadjusted models, adjustment for demographic confounders (models 1; figure 3) attenuated the effect for all disability domains, but all HRs remained above one. The 95\% CIs for models adjusted for age, town, living status and SES (model 1) overlap with those for the unadjusted model (model 0 ) in all disability domains.

Further adjustment for lifestyle factors, health conditions and prior disability domains in model 2 led to attenuation of the mortality effect in all domains (figure 3), but again all effects remained above one (figure 3 ). For example, after adjustment for potential confounders, lifestyle factors and health conditions mortality hazard increased by 1.08 (95\% CI 1.03 to 1.13 ) per unit increase in impairment score.

However, impairments were no longer associated with elevated mortality after adjusting for basic and complex activities and participation (model 3; $\mathrm{HR}=0.97,95 \% \mathrm{CI} 0.91$ to 1.03 ). Similarly, basic activities were also no longer associated with mortality after adjusting for complex activities and participation (model 3; figure 3) or all domains and confounders (model 4; figure 3).

Conversely, complex activities remained a predictor of elevated mortality after adjustment for participation (model 3; $\mathrm{HR}=1.08$, 95\% CI 1.02 to 1.15 ) and for all domains and confounders (model 4; HR=1.09, 95\% CI 1.01 to 1.18). Participation also remained an independent predictor of mortality following adjustment for all dimensions and confounders (model 4; $\mathrm{HR}=1.10,95 \%$ CI 1.04 to 1.16 ).

\section{Sensitivity analyses}

Evidence suggests that non-responders to surveys are more likely to be in ill health. ${ }^{33}{ }^{34}$ Therefore, models were re-estimated adopting a more extreme scenario whereby all missing data were

Table 2 Baseline response profile and mean scores by alive or dead status at follow-up

\begin{tabular}{|c|c|c|c|c|c|c|c|c|}
\hline & \multicolumn{4}{|l|}{ Alive } & \multicolumn{4}{|l|}{ Dead } \\
\hline & Responders & $\%$ & Mean & SD & Responders & $\%$ & Mean & SD \\
\hline Age & 3478 & 100 & 71.11 & 5.37 & 653 & 100 & 74.63 & 5.20 \\
\hline SES & 2906 & 84 & 4.18 & 2.14 & 513 & 79 & 4.70 & 2.29 \\
\hline Lifestyle score & 3375 & 97 & 5.43 & 2.34 & 623 & 95 & 6.37 & 2.33 \\
\hline CV disease score & 3479 & 100 & 0.71 & 1.18 & 653 & 100 & 1.11 & 1.54 \\
\hline Non-CV disease score & 3479 & 100 & 1.45 & 1.58 & 653 & 100 & 1.93 & 2.05 \\
\hline Impairment score & 3194 & 92 & 2.51 & 2.07 & 505 & 77 & 3.37 & 2.32 \\
\hline Basic activities score & 3194 & 92 & 2.32 & 2.65 & 505 & 77 & 3.84 & 3.15 \\
\hline Complex activities score & 3185 & 92 & 1.51 & 2.25 & 502 & 77 & 3.06 & 3.06 \\
\hline Participation score & 3151 & 91 & 1.28 & 2.35 & 493 & 75 & 2.77 & 3.14 \\
\hline Living alone & 3143 & 90 & $35 \%$ & & 493 & 75 & $48 \%$ & \\
\hline
\end{tabular}

CV, cardiovascular; SES, socioeconomic status. 
HR of the different dimensions with different adjustments

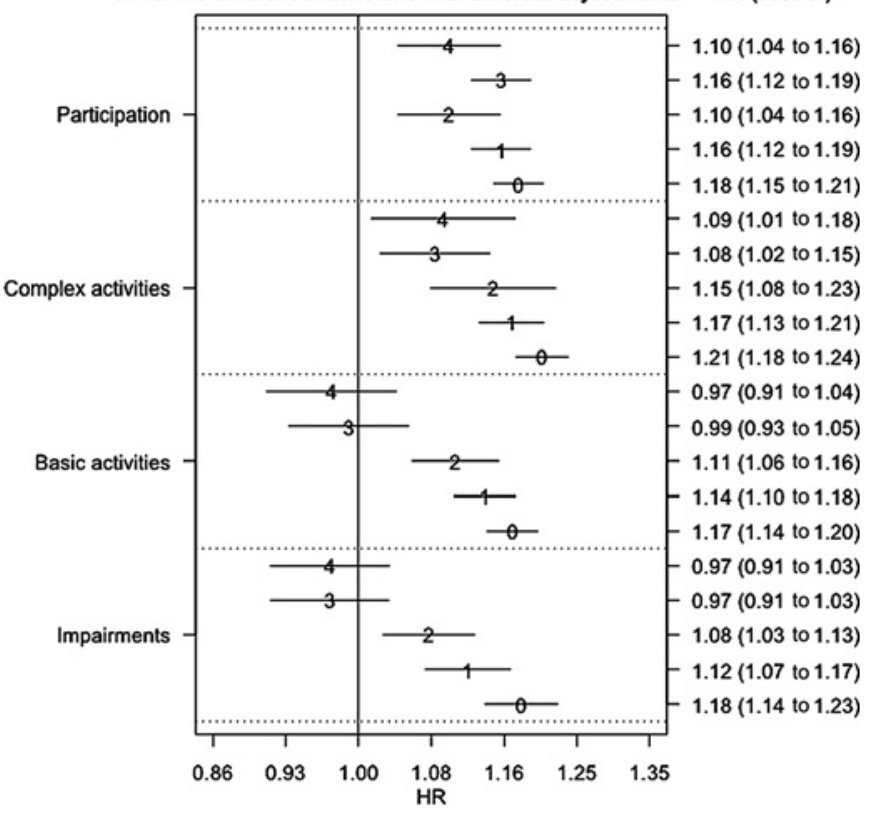

0 : Crude

1: Adjusted for age, town, living status and SES

2: Adjusted for age, town, living status, SES and prior domains

3: Adjusted for age, town, living status, SES and subsequent domains

4: Adjusted for age, town, living status, SES and all domains

Figure 3 Mortality HRs per unit of disability domain score, with cumulative adjustment.

assumed to be adverse in the construction of domain scores. The same pattern of association with mortality with sequential adjustments was observed (figure 4). This suggests that it is unlikely that the relative effect of the disability domains on mortality is explained by differential missing data in the domain scores. Under the extreme missing data scenario, there is weaker evidence for difference in the hazard of dying per unit increase in participation score (model 4; figure 4), while under the original missing data scenario, there was weaker evidence per unit increase in complex activities score (model 4; figure 3).

In further sensitivity analyses, health conditions were entered separately into the models rather than as a cumulative domain score. This led to more imprecise estimates resulting from the loss of sample size but no substantive change in results compared with the models using the domain scores for health conditions.

\section{DISCUSSION}

This study provides evidence that all ICF disability domains are independent predictors of mortality after controlling for lifestyle factors and health conditions in a nationally representative sample of British women. However, after mutual adjustment for other disability domains, health conditions and lifestyle factors, only complex activities and participation are independent predictors of mortality. Thus, the ICF disability domains most closely association with death have been pinpointed as social participation and complex activities. At face value, these findings are consistent with the social model of disability, suggesting that, at least in terms of mortality, the ability of a woman to perform complex activities or participate in her social environment may be more important than her biological impairments or basic activities limitations.

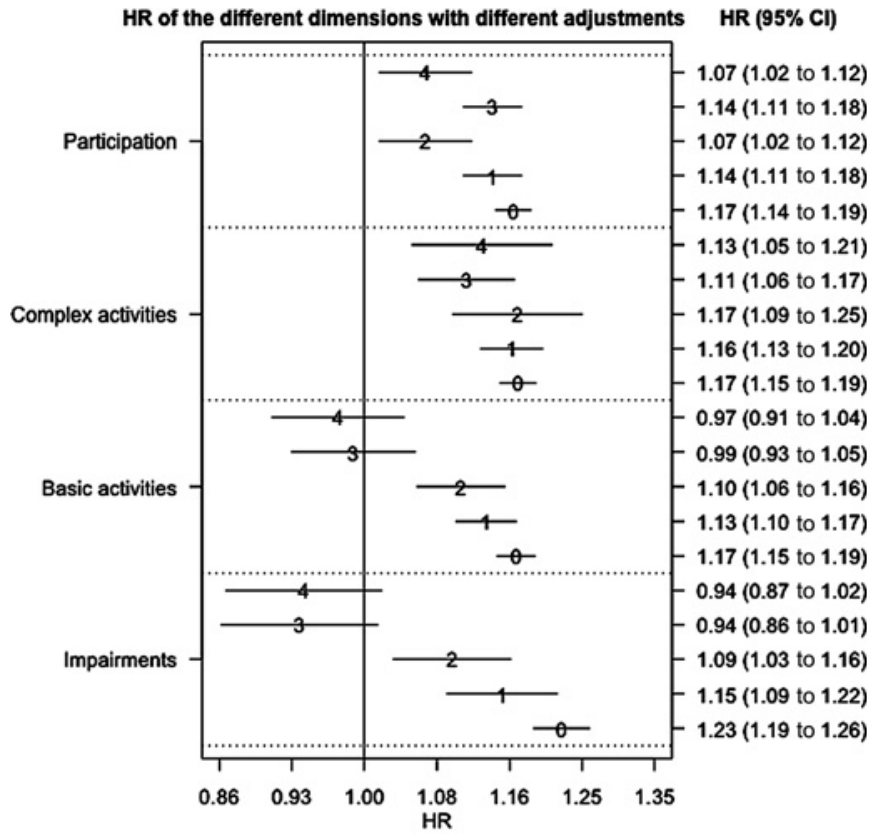

0 : Crude

1: Adjusted for age, town, living status and SES

2: Adjusted for age, town, living status, SES and prior domains

3: Adjusted for age, town, living status, SES and subsequent domains

4: Adjusted for age, town, living status, SES and all domains

Figure 4 Mortality HRs per unit of disability domain score, with cumulative adjustment (assuming missing data are all adverse).

The independent effect of participation on mortality is consistent with a previous finding of social engagement on cardiovascular mortality in British men (HR 0.70, 95\% CI 0.53 to 0.93). ${ }^{35}$ Similarly, a $\mathrm{HR}=0.87$ (95\% CI 0.77 to 0.88 ) for social activities was found in a population of elderly people living in East London or Essex. By comparison, the strength of association in the current study is relatively modest at approximately a $10 \%$ increase in the hazard of death per unit increase in participation (or complex activities) score. However, direct comparison of effect sizes across studies is hindered by the use of different survey instruments and methods in different studies. This impact of measurement has been highlighted in a recent metaanalysis, where stronger effects were found with multidimensional assessments of social integration such as the one in the current study. ${ }^{20}$ This meta-analysis found an $\mathrm{OR}=1.91$ (95\% CI 1.63 to 2.23 ) for survival with complex measures of social integration. ${ }^{20}$ However, estimates with the least statistical control for confounding and the greatest level of contrast were meta-analysed, both likely to reveal stronger effects compared with methods applied in the current study. ${ }^{20}$ Furthermore, effect sizes in the current study relate to a per unit increase in ICF domain score, in other words the additional mortality risk associated with the addition of a single extra limitation or restriction. This compares with the Ramsay et al ${ }^{35}$ study using a nine item scale where HRs compared risk in those scoring 8-9 versus $0-3$.

Low social engagement was also associated with higher mortality after controlling for physical health in a small Nottingham city sample. ${ }^{36}$ In a Dublin city sample, higher social engagement has been associated with enhanced mental and physical health, cognition and quality of life. ${ }^{37}$ Internationally, loneliness has been associated with higher mortality risk in a Finnish population sample ${ }^{38}$; while the less socially engaged 
were found to be less healthy in a large Dutch community sample. ${ }^{39}$ Strong cross-sectional association between social participation and disability were also found in a US community sample, but there was no evidence that participation was protective against functional decline, suggesting that findings may be more consistent with reciprocal causation, that is, functional decline causes lower participation levels. ${ }^{40}$

Our finding with respect to complex activities replicates the established evidence for an association between activities of daily living or instrumental activities of daily living and mortality. ${ }^{41-43}$ However, an interesting finding in the current study is that evidence for an independent association was limited to 'complex' activities, while 'basic' activities were not found to have an independent association with mortality.

\section{Measurement issues}

'Reverse causality' can arise through the presence of morbidities contributing to both future mortality and disability at baseline measurement. While confounding by health conditions was controlled for in the analyses, it is possible that this control was incomplete due to measurement error in the diagnosis of health conditions. Furthermore, despite the rich phenotyping available in the BWHHS, it was still only possible to control for a limited number of conditions and lifestyle behaviours. In addition, health conditions and lifestyle behaviours were modelled as binary variables, and therefore, potentially important variability in the severity of these conditions was not accounted for. Indeed, it is quite possible that the disability domains studied were acting as proxies for severity of health conditions.

The BWHHS questionnaire was not designed to assess the ICF and hence coverage of some domains may be incomplete. Impairments in particular were measured through self-report only and may be under-represented. Another measurement problem relates to the potential correlation between ICF domains. For example, locomotor activity limitation has previously been shown to be strongly associated with reduced participation in the BWHHS. ${ }^{25}$ Attempts have been made to identify measures of impairment, activity and participation uncontaminated by other ICF domains for particular health outcomes, such as the Aberdeen IAP for joint replacement surgery. ${ }^{44}$ It is likely that some items included in the current study may not purely measure the domain in question, thereby leading to misclassification bias and potential underestimation of domain effects.

Another potential limitation stems from partial questionnaire completion resulting in missing data in the disability domains. A lower level of response was found among those who subsequently died compared with those who were alive at the end of follow-up. It is likely that non-responders may have had a higher disability load as well as being more likely to die, and therefore, complete case estimates of the association between disability and death will be conservative. However, in sensitivity analyses, results were found to be robust to the more extreme missing data scenario where all missing disability data were modelled as adverse.

The BWHHS is a prospective cohort of elderly women in Great Britain; therefore, results may not be generalisable to other populations with different disability or mortality profiles.

\section{Implications of a social participation focus}

For the association between social participation and mortality to be considered causal, identification of a biologically plausible pathway would be required. It has been argued that a direct pathway may exist through physiological changes to the cardiovascular, hormonal and immunological systems. ${ }^{45}$ Social support has been linked to favourable inflammatory profiles in men, ${ }^{46} 47$ as well as to enhanced immune response and increased resistance to upper respiratory illness. ${ }^{48} 49$ An indirect pathway may operate through encouraging healthier lifestyle behaviours, such as improved diet and greater physical activity. Yet lifestyle factors were controlled for in the current study, albeit imperfectly. Furthermore, social activities that have little physical fitness benefit have also been shown to be associated with survival benefits. ${ }^{50}$ Another possible pathway is via psychosocial benefits. Social engagement has been prospectively associated with favourable change in depressive symptoms in some populations. ${ }^{51} 52$ There is also some evidence for lower cognitive decline in those with more social ties, ${ }^{53}$ while social integration has been found to delay memory loss in the US Health and Retirement Study. ${ }^{54}$ However, it is difficult to rule out the possibility of residual confounding by unmeasured health status in such associations.

\section{Public health implications}

Inability to carry out complex activities and limited social participation predict survival prospects and could provide a means of identifying 'at risk' women in the community for targeted support. If association with social participation is causal, this would suggest that interventions to improve participation in older people could delay mortality. However, complex care interventions have previously been found to have no overall benefit for mortality, although they can help with independent living. ${ }^{55}$ This is consistent with the recent finding that received social support is less predictive than social integration for mortality. ${ }^{20}$ Hence, community-based interventions that leverage naturally occurring community social capital with targeted individual interventions to increase social participation may prove more effective for reducing mortality. Conversely, in the current climate of worldwide recession, cuts to public spending could precipitate a decline in social participation among the elderly people that might ultimately result in higher mortality.

\section{What is already known on this subject}

- The proportion of older disabled people in the UK and many other high-income countries is increasing rapidly.

- Different aspects of disability, often studied in isolation, have been associated with mortality. However, their relative importance and whether their effect is accounted for by lifestyle factors and comorbidities are not well understood.

\section{What this study adds}

- Using the disability framework provided by the WHO's International Classification of Functioning, Disability and Health, we showed that social participation and complex activities were the strongest predictors of mortality. These associations were independent of other disability domains as well as from lifestyle factors and major chronic comorbidities.

- This study demonstrates the importance of social participation and complex activities in the elderly people, highlighting that these should not be overlooked in planned reforms to the social care system. 
Funding The British Women Heart and Health Study is supported by grants from the British Heart Foundation (EPNCCD08) and the Department of Health (009/0049). The funders had no role in the study design; in the collection, analysis and interpretation of the data; in the writing of the report or in the decision to submit the paper for publication.

\section{Competing interests None.}

Ethics approval This study was approved by London School of Hygiene \& Tropical Medicine Ethics Committee and 23 Local Research Ethics Committees.

Contributors All authors contributed to research design, interpreting data and drafts of the paper. DP performed the statistical analyses.

Provenance and peer review Not commissioned; externally peer reviewed.

\section{REFERENCES}

1. Office for National Statistics. Statistical Bulletin. National population projections, 2008-based. 21 October 2009. Available online at http://www.ons.gov.uk/ons/ taxonomy/index.html?nscl=Population + Projections

2. Malley J, Wittenberg R, Comas-Herrera A, et al. Long-term care expenditure for older people, projections to 2022 for Great Britain. In: The Personal Social Services Research Unit (PSSRU), ed. Report to the Institute for Public Policy Research (IPPR). 2005.

3. Fries JF. Aging, natural death, and the compression of morbidity. N Engl J Med 1980;303:130-5.

4. Secretary of State for Work and Pensions. Building a Society for All Ages. Secretary of State for Work and Pensions, 2009.

5. Department of Health. Speech by the Rt Hon Alan Johnson MP, Secretary of State for Health, 21 May 2008: Prevention Speech: Old Age is the New Middle Age. King's Fund. http://webarchive.nationalarchives.gov.uk/+/www.dh.gov.uk/en/MediaCentre/ Speeches/DH_085020

6. Secretary of State for Health HM Government. Healthy Lives, Healthy People: Our strategy for Public Health in England. Secretary of State for Health HM Government, 2010.

7. Graham P, Blakely T, Davis P, et al. Compression, expansion, or dynamic equilibrium? The evolution of health expectancy in New Zealand. J Epidemiol Community Health 2004;58:659-66.

8. Pillai R, Rankin J, Stanley K, et al. Disability 2020: opportunities for the full and equal citizenship of disabled people in Britain in 2020. In: ippr trading Itd. for the Disability Rights Commission. 2007.

9. Fries JF. Measuring and monitoring success in compressing morbidity. Ann Intern Med 2003;139:455-9

10. Office for National Statistics. Office for National Statistics. Health expectancies at birth and at age 65 in the United Kingdom 2000-02 to 2006-08. 24 February 2010. Table 1. Available online at http://www.ons.gov.uk/ons/taxonomy/index.html? nscl $=$ Disability + and + Self-reported + Health

11. Jagger C, Matthews RJ, Matthews FE, et al. Cohort differences in disease and disability in the young-old: findings from the MRC Cognitive Function and Ageing Study (MRC-CFAS). BMC Public Health 2007:7:156.

12. Christensen K, Doblhammer G, Rau R, et al. Ageing populations: the challenges ahead. Lancet 2009;374:1196-208.

13. Bebbington A, Darton R. Healthy Life Expectancy in England and Wales: Recent Evidence. The Personal Social Services Research Unit (PSSRU) Discussion Paper 1205. 1996.

14. Shakespeare T, Wastson N. Defending the social model. Disabil Soc 1997:12:293-300.

15. World Health Organization. International Classification of Functioning, Disability and Health. Geneva: World Health Organization, 2001.

16. Pollard B, Johnston M, Dieppe P. What do osteoarthritis health outcome instruments measure? Impairment, activity limitation, or participation restriction? J Rheumatol 2006;33:757-63.

17. Gale CR, Martyn CN, Cooper C, et al. Grip strength, body composition, and mortality. Int J Epidemiol 2007:36:228-35.

18. Newman AB, Simonsick EM, Naydeck BL, et al. Association of long-distance corridor walk performance with mortality, cardiovascular disease, mobility limitation, and disability. JAMA 2006;295:2018-26.

19. Cacciatore $\mathbf{F}$, Abete $\mathrm{P}$, Maggi $\mathrm{S}$, et al. Disability and 6-year mortality in elderly population. Role of visual impairment. Aging Clin Exp Res 2004:16:382-8.

20. Holt-Lunstad J, Smith TB, Layton JB. Social relationships and mortality risk: a metaanalytic review. PLoS Med 2010;7:e1000316.

21. Euro0ol Group. EQ-5D A standardised instrument for use as a measure of health outcome. EuroQol Group.

22. Hunt SM, McKenna SP, Williams J. Reliability of a population survey tool for measuring perceived health problems: a study of patients with osteoarthrosis. $J$ Epidemiol Community Health 1981;35:297-300.

23. Katz S. Assessing self-maintenance: activities of daily living, mobility, and instrumental activities of daily living. J Am Geriatr Soc 1983;31:721-7.

24. WHO. ICF Browser. WHO, 2010.
25. Adamson J, Lawlor DA, Ebrahim S. Chronic diseases, locomotor activity limitation and social participation in older women: cross sectional survey of British Women's Heart and Health Study. Age Ageing 2004;33:293-8.

26. Ebrahim S, Wannamethee G, McCallum A, et al. Marital status, change in marita status, and mortality in middle-aged British men. Am J Epidemiol 1995; 142:834-42.

27. Fyrand $\mathbf{L}$, Moum T, Finset $A$, et al. The impact of disability and disease duration on social support of women with rheumatoid arthritis. J Behav Med 2002 25:251-68.

28. Adamson J, Hunt K, Ebrahim S. Socioeconomic position, occupational exposures, and gender: the relation with locomotor disability in early old age. J Epidemiol Community Health 2003:57:453-5.

29. Ebrahim S, Papacosta O, Wannamethee G, et al. Social inequalities and disability in older men: prospective findings from the British regional heart study. Soc Sci Med 2004:59:2109-20.

30. Adamson JA, Ebrahim S, Hunt K. The psychosocial versus material hypothesis to explain observed inequality in disability among older adults: data from the West of Scotland Twenty-07 Study. J Epidemiol Community Health 2006;60:974-80.

31. Ebrahim S, Adamson J, Ayis S, et al. Locomotor disability: meaning, causes and effects of interventions. J Health Serv Res Policy 2008:(13 Suppl 3):38-46.

32. Lawlor DA, Davey Smith G, Patel R, et al. Life-course socioeconomic position, area deprivation, and coronary heart disease: findings from the British Women's Heart and Health Study. Am J Public Health 2005;95:91-7.

33. Rockwood K, Stolee P, Robertson D, et al. Response bias in a health status survey of elderly people. Age Ageing 1989;18:177-82.

34. Hoeymans N, Feskens EJ, Van Den Bos GA, et al. Non-response bias in a study of cardiovascular diseases, functional status and self-rated health among elderly men Age Ageing 1998:27:35-40.

35. Ramsay S, Ebrahim S, Whincup P, et al. Social engagement and the risk of cardiovascular disease mortality: results of a prospective population-based study of older men. Ann Epidemiol 2008;18:476-83.

36. Bennett KM. Low level social engagement as a precursor of mortality among people in later life. Age Ageing 2002:31:165-8.

37. Golden J, Conroy RM, Lawlor BA. Social support network structure in older people: underlying dimensions and association with psychological and physical health. Psychol Health Med 2009;14:280-90

38. Tilvis RS, Laitala V, Routasalo PE, et al. Suffering from loneliness indicates significant mortality risk of older people. J Aging Res 2011;2011:534781.

39. Croezen S, Haveman-Nies A, Alvarado VJ, et al. Characterization of different groups of elderly according to social engagement activity patterns. J Nutr Health Aging 2009;13:776-81.

40. Mendes de Leon CF, Glass TA, Berkman LF. Social engagement and disability in a community population of older adults: the New Haven EPESE. Am J Epidemiol 2003;157:633-42.

41. Bowling A, Grundy E. Differentials in mortality up to 20 years after baseline interview among older people in East London and Essex. Age Ageing 2009;38:51-5.

42. Bowling A. Predictors of mortality among a national sample of elderly widowed people: analysis of 28-year mortality rates. Age Ageing 2009;38:527-30.

43. Scott WK, Macera CA, Cornman CB, et al. Functional health status as a predictor of mortality in men and women over 65. J Clin Epidemiol 1997:50:291-6.

44. Pollard B, Dixon D, Dieppe P, et al. Measuring the ICF components of impairment activity limitation and participation restriction: an item analysis using classical test theory and item response theory. Health Qual Life Outcomes 2009:7:41.

45. Uchino BN. Social support and health: a review of physiological processes potentially underlying links to disease outcomes. J Behav Med 2006;29:377-87.

46. Loucks EB, Sullivan LM, D'Agostino RB Sr, et al. Social networks and inflammatory markers in the Framingham Heart Study. J Biosoc Sci 2006;38:835-42.

47. Ford ES, Loucks EB, Berkman LF. Social integration and concentrations of C-reactive protein among US adults. Ann Epidemiol 2006;16:78-84.

48. Lutgendorf SK, Sood AK, Anderson B, et al. Social support, psychological distress, and natural killer cell activity in ovarian cancer. J Clin Oncol 2005:23:7105-13.

49. Cohen S, Doyle WJ, Skoner DP, et al. Social ties and susceptibility to the common cold. JAMA 1997:277:1940-4

50. Glass TA, de Leon CM, Marottoli RA, et al. Population based study of social and productive activities as predictors of survival among elderly Americans. BMJ 1999:319:478-83.

51. Glass TA, De Leon CF, Bassuk SS, et al. Social engagement and depressive symptoms in late life: longitudinal findings. J Aging Health 2006;18:604-28.

52. Isaac V, Stewart R, Artero $\mathrm{S}$, et al. Social activity and improvement in depressive symptoms in older people: a prospective community cohort study. Am J Geriatr Psychiatry 2009;17:688-96.

53. Bassuk SS, Glass TA, Berkman LF. Social disengagement and incident cognitive decline in community-dwelling elderly persons. Ann Intern Med 1999;131:165-73.

54. Ertel KA, Glymour MM, Berkman LF. Effects of social integration on preserving memory function in a nationally representative US elderly population. Am J Public Health 2008;98:1215-20.

55. Beswick AD, Rees K, Dieppe P, et al. Complex interventions to improve physical function and maintain independent living in elderly people: a systematic review and meta-analysis. Lancet 2008;371:725-35. 